\title{
Thermotherapy and Vibroacoustic Therapy in Preventing and Possibly Reversing Dementia and Alzheimer's
}

\section{George Grant ${ }^{1 *}$ and Jay P Vanden Heuvel ${ }^{2}$}

${ }^{1}$ Professor, Founder of www.academyofwellness.com, Toronto, ON, Canada

${ }^{2}$ Professor, www.naturalselections.net, Green Bay Wisconsin, USA

*Corresponding Author: George Grant, Professor, Founder of www.academyofwellness.com, Toronto, ON, Canada.

DOI: 10.31080/ASNE.2020.03.0206
Received: June 10, 2020

Published: July 30, 2020

(C) All rights are reserved by George Grant and Jay P Vanden Heuvel.

\begin{abstract}
The Objective of this Literature Review is to find any published scientific evidence to correlate the use of both Thermotherapy [Hyperthermia][Heat Therapy] and Vibroacoustic therapy [Vibration + Sound] to prevent and possibly reverse chronic neurological conditions like Dementia and Alzheimer's Disease.
\end{abstract}

Keywords: Thermotherapy; Vibroacoustic Therapy; Alzheimer's Diseases

\section{Introduction}

Neurodegenerative diseases involve the progressive deterioration of structures within the central nervous system responsible for motor control, cognition, and autonomic function. Dementia, Alzheimer's disease and Parkinson's disease are among the most common neurodegenerative disease and have an increasing prevalence over the age of 50. Central in the pathophysiology of these neurodegenerative diseases is the loss of protein homeostasis, resulting in misfolding and aggregation of damaged proteins. An element of the protein homeostasis network that prevents the dysregulation associated with neurodegeneration is the role of molecular chaperones. Heat shock proteins (HSPs) are chaperones that regulate the aggregation and disaggregation of proteins in intracellular and extracellular spaces, and evidence supports their protective effect against protein aggregation common to neurodegenerative diseases. Consequently, upregulation of HSPs, such as HSP70, may be a target for therapeutic intervention for protection against neurodegeneration. A novel therapeutic intervention to increase the expression of HSP may be found in heat therapy and/or heat acclimation. In healthy populations, these interven- tions have been shown to increase HSP expression. Elevated HSP may have central therapeutic effects, preventing or reducing the toxicity of protein aggregation, and/or peripherally by enhancing neuromuscular function. Broader physiological responses to heat therapy have also been identified and include improvements in muscle function, cerebral blood flow, and markers of metabolic health. These outcomes may also have a significant benefit for people with neurodegenerative disease. While there is limited research into body warming in patient populations, regular passive heating (sauna bathing) has been associated with a reduced risk of developing neurodegenerative disease. Therefore, the emerging evidence is compelling and warrants further investigation of the potential benefits of heat acclimation and passive heat therapy for sufferers of neurodegenerative diseases.

Richway International [Fuji Bioscience] introduced recently The BioAcoustic Mat ${ }^{\mathrm{TM}}$ which is a revolutionary vibroacoustic therapy device made by Richway and Fuji Bio, makers of the worldrenowned Biomat. We will be conducting a clinical trial using this new device on 12 subjects suffering from Dementia from our clinics in Canada and US. 
Years of scientific research has shown that specific low frequency sound pulsations and beautiful brainwave entraining music have powerful therapeutic effects on our health and well-being. The practical applications of sound being used in treatments have been present in cultures throughout our history. Early cultures such as the Aborigines, a tribal group from Australia, have used sound to heal the ill. They would use a yidaki, a musical instrument in which blown air would create very low vibrations.

The BioAcoustic Mat ${ }^{\mathrm{TM}}$ employs a powerful low frequency sound generator that delivers a deep acoustic massage for joints and tissues. The music pieces on the BioAcoustic Mat ${ }^{\mathrm{TM}}$ apply the low frequencies of 30-68 Hz to massage large muscles and the higher 52$88 \mathrm{~Hz}$ level for smaller muscles.
Dr. Lee Bartel, the world's foremost authority on vibroacoustic therapy's research has shown scientifically developed sounds will relax and renew your body naturally. The high-fidelity upper speakers add another dimension of sound therapy to the BioAcoustic Mat $^{\mathrm{TM}}$. The beautiful music on these upper speakers have been designed to provide a calming sense of well-being.

Drawing on years of experience, Dr. Bartel has created tracks that get you ready for sleep by stimulating deep sleep delta brainwaves, helps you relax by stimulating alpha brainwaves, or helps you get energized and solve problems by stimulating your theta brainwaves.

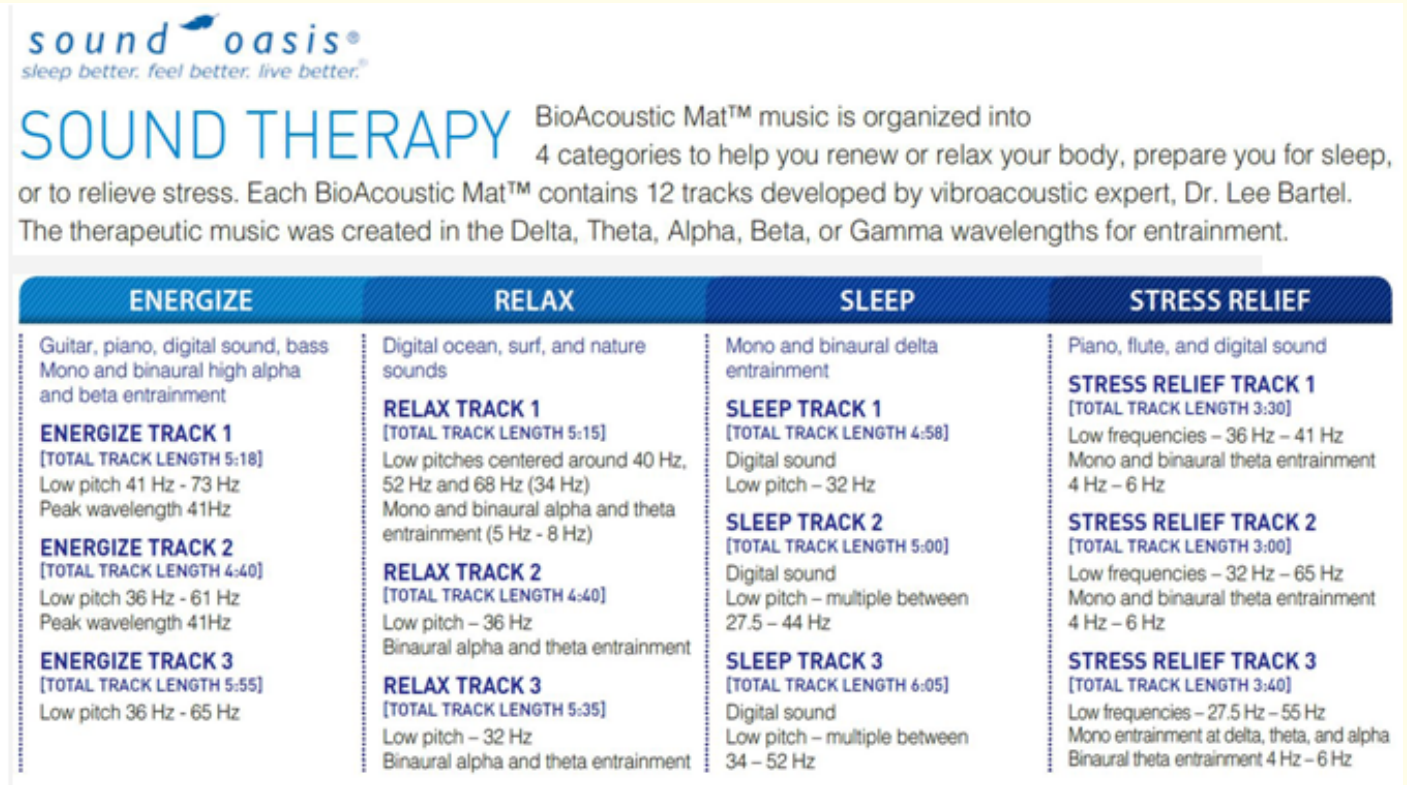

\section{Four categories of music ENERGIZE + RELAX + DEEP SLEEP + RELIEVE STRESS}

Four categories and 12 therapeutic tracks

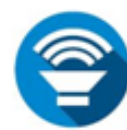

SOUND THERAPY

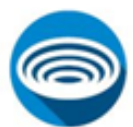

VIBRATION THERAPY

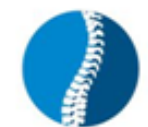

BODY CARE

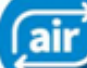

COMFORT

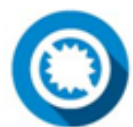

EASY TO CLEAN

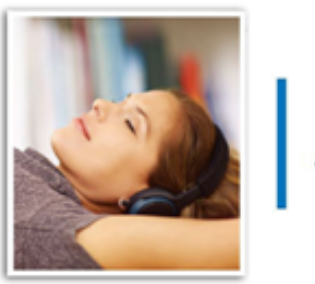

\section{REBOOT AND RESTORE WITH THERAPEUTIC SOUNDS AND VIBRATIONS}

Figure a 
Feel the pulses of the BioAcoustic Mat's ${ }^{\mathrm{TM}}$ healing energy.

Vibroacoustic relaxation is not just for the brain. It also provides deep physical cellular stimulation to the skin, muscles, and joints.

Research has shown that relaxing music helps reduce stress. However, with the BioAcoustic Mat ${ }^{\mathrm{T}}$, you not only hear the music but feel it as well. Like a massage, the vibrations from the BioAcoustic Mat ${ }^{\mathrm{TM}}$ relaxes you in two ways.

First, powerful low frequency speakers and transducers are used to help deliver a deep acoustic cellular massage. The lower frequencies are used to massage large muscles, and the higher frequencies for smaller muscles. Secondly, these sounds affect brain activity to help contribute to a state of mental well-being. Professors and researchers have collaborated to create beautiful soothing music which is designed to help you reach a calming sense of tranquility. The music is composed and designed in a way to complement the frequencies emitted by the speakers. The vibrations stimulate the nerves in the spine, brain stem, and limbic system that drives emotional response, and activates the auditory nerves that connect to your muscle tissue, and the low frequency bass causes the muscle tissue to be at ease.

Central in the pathophysiology of neurodegenerative diseases is the loss of protein homeostasis and the progressive loss of selective neurons. Protein homeostasis involves a complex system of protein synthesis, folding, disaggregation, and degradation that ensures the correct function of the human body and particularly the central nervous system [24]. Loss of protein homeostasis, due to protein mis-folding and aggregation of damaged proteins, is a hallmark of neurodegenerative diseases such as Alzheimer's and Parkinson's diseases [25]. Alzheimer's and Parkinson's are the two most common degenerative neurological conditions and are more prevalent with advancing age. Both of these neurodegenerative diseases are progressive with pathological features demonstrating topographic distribution. The progressive loss of selective neurons includes amyloidosis, tauopathies, alpha-synucleinopathies, and proteinopathies, all of which have their own characteristic histopathological imaging features, as well as clinical symptomology. The diseases are incurable and result in long-term cognitive, psychological, motor, and non-motor impairments that have a profound impact on functional mobility, psychological well-being, independent living, and quality of life.

\section{Alzheimer's disease}

Alzheimer's disease is the most prevalent neurodegenerative disease and is the most common form of dementia [30], which affects 40-50 million people worldwide [41]. Early stages of Alzheimer's presents with mild cognitive impairment involving memory loss and progresses with deficits in attention, language, and visuospatial abilities [41]. Social withdrawal accompanies disease progression, as symptoms include a reduced capacity to perform activates of daily living, impaired executive function and judgment, along with disorientation [5]. These outcomes have a significant impact on independence, quality of life, and years of life with a disability [7]. Furthermore, the economic cost of dementia is $\$ 968$ billion globally (Garman., et al). These costs are born by individuals and their caregivers, social health services, as well as public and private health care providers [25]. Due to the aging population, the prevalence and impact of Alzheimer's disease are anticipated to increase in the future [21].

Neurodegenerative diseases such as Alzheimer's are marked by a loss of cellular protein homeostasis [26]. The pathophysiology of Alzheimer's is evidenced by intracellular and extracellular amyloid- $\beta$ plaques as well as neurofibrillary tangles of hyperphosphorylated tau [26]. Neurodegeneration occurs as a result of the accumulation of tau proteins and atrophy of cerebral cortices. Amyloid deposits occur in the neocortex and hippocampus (Phases 1 and 2), the striatum (Phase 3), the brainstem (Phase 4), and the cerebellum (Phase 5) [16]. In concert with abnormal protein accumulation, the pathogenesis of Alzheimer's disease may also involve vascular impairments leading to chronic cerebral hypoperfusion $[5,30]$ combat these pathophysiological progressions, therapeutic interventions to improve protein quality control and regulation or improve vascular health and function have been recommended $[5,26]$.

\section{Heat shock proteins as a therapeutic target}

Recent reviews have clearly identified the upregulation of HSPs as thermally activated therapeutic targets for the treatment of neurodegenerative diseases including Parkinson's and Alzheimer's $[24,26]$. HSPs are a collective family of proteins, suffixed by their molecular weight (in kilodaltons; $\mathrm{kDa}$ ), which are present in both constitutively expressed, and inducible isoforms across several intracellular tissue sites and in extracellular fluid following stress [27]. Relative to increased intracellular HSP content (a necessary component for protective cellular adaptation), the pres-

Citation: George Grant and Jay P Vanden Heuvel. "Thermotherapy and Vibroacoustic Therapy in Preventing and Possibly Reversing Dementia and Alzheimer's". Acta Scientific Neurology 3.8 (2020): 24-36. 
ence of extracellular changes in HSP concentration reflects a less pertinent (in the context of adaptation) transient stress response which acts as an acute signaling response. The $70 \mathrm{kDa}$ (HSPA) and $90 \mathrm{kDa}$ (HSPC) family of HSPs, hereafter referred to as HSP70 and HSP90, are generally the most widely studied responders to thermal stressors and are likely of most relevance within the field of heat therapy and heat adaptation for neurodegenerative disease [28]. HSP70 and HSP90 function as chaperones to ensure appropriate cell function and have distinct roles in the unfolded protein response, e.g., recognizing misfolded or mis-localized proteins that may be subsequently degraded by the proteasome, and are a key component of chaperone-mediated autophagy [6]. It is outside of the focus of this review to describe each of these roles, with the reader directed elsewhere to contextualize these actions [6]. As therapeutic targets, HSP70 and HSP90 may be considered to have a direct and indirect role in neurodegenerative diseases. Direct roles for HSPs on the nervous system arise from the aforementioned notion that aggregation of misfolded proteins is characteristic of neurodegenerative diseases, including Parkinson's, Alzheimer's, and Huntington's [7]. In Parkinson's, HSP70 is reported as being of decreased gene expression [8]. While during proteomic profiling, reduced phosphorylation of HSP90 is also reported [6]. Pharmacological and animal models utilizing HSP expression (elevated HSP70 and reduced HSP90) have reduced the aggregation and toxicity of alpha-synuclein in Parkinson's disease [8]. In Alzheimer's, HSP70 may suppress the proteolysis of amyloid precursor protein (Danzer., et al.) and in addition to HSP70, HSP90, and small HSPs reduce the formation of A-beta fibrils and A-beta toxicity which subsequently form amyloid plaques. Tauopathy occurrence in Alzheimer's may also be positively impacted by HSP changes with HSP70 and HSP90. Further, HSPs have been found to regulate huntingtin via reduced cell aggregation in Huntington's disease (Brunt., et al.), and slows the muscle denervation of amyotrophic lateral sclerosis (Motor Neuron Disease) [21].

Much of the literature describing these responses involve complex and isolated tissue/cell models to understand how HSP manipulation impacts upon neurodegenerative disease factors, thus direct application for humans remains unknown. However, with mechanistic support for the role of HSP augmentation to improve disease states, the application of heat therapy and/or heat adaptation in this context warrants further investigation.
Responses to active (Exercise Heat Acclimation) and passive heat therapy in healthy adults

Physical activity and exercise have long been identified as mechanisms of inducing physiological stressors and subsequent positive adaptations in healthy and chronic disease [15] populations. Unfortunately, those with increasing disease severity or diseases that challenge their motor control capabilities may be physically incapable of performing such beneficial exercise. Heat therapy has recently been targeted as a potential vehicle to evoke these positive thermal-induced adaptations in those precluded from undertaking exercise. Experimental investigations, large cohort surveys and reviews have expressed the potential for passive heating to improve physical and mental health in patients with cardiovascular disease [17], diabetes [34], peripheral arterial disease [4], and depression [40].

While there is a myriad of beneficial physiological and molecular effects of active and passive heating, this review will primarily focus specifically on the outcome of HSP expression, for its potential to influence proteostasis in neurodegenerative disease. For active and passive heating to be effective in increasing HSP expression, the minimum exposure requirements to elicit a desirable response in HSPs, from both acute and chronic (repeated) exposure, needs to be identified.

Acute effects of body warming on heat shock proteins

Transcription of HSP mRNA, an essential step before protein translation, is primarily regulated by Heat shock factor protein 1 (HSF-1) as part of the Heat Shock Response [28]. HSF-1 activation involves a complex series of regulatory events, including nuclear localization, oligomerization and acquisition of HSE-DNA binding, ultimately resulting in the transcription of HSP mRNA in response to the thermal and physiological challenge [14]. Sufficient mRNA transcription then leads to increased protein within the stressed cell.

Precise parameters for intracellular increases, and thus cellular adaptation, have been less clearly defined. For example, mean core body temperature may not be the sole marker of an increase, rather the rate of change in core body temperature may be of greater importance to signal HSF-1 to HSP70 pathways. In the more common exercise-heat stress model, a recent analysis concluded that when transcription of the related HSP70 and HSP90 $\alpha$ mRNA is important, protocols should rapidly induce large, prolonged changes 
in core body temperature [43]. This notion was supported by evidence that, when analyzed collectively, significant predictors of the post-exercise change in HSP70 and HSP90 $\alpha$ mRNA were the change in mean and peak core body temperature, and the duration core body temperature was $\geq 38.5^{\circ} \mathrm{C}$ [44]. It should be acknowledged that these data describe responses to exercise-heat stress, an intervention that is likely to be challenging to implement in clinical populations. Accordingly, passive heating via body warming may prove to be a more efficacious intervention.

The HSP response to localized or whole body warming has also been investigated. In passive heating models, increases in HSP70 and HSP90 mRNA have been evidenced as peaking $30 \mathrm{~min}$ following 90 min of local heating to either the thigh or the whole leg of healthy human volunteers (Gao., et al.) Regrettably, no intramuscular temperature data is available from this experiment to assist with identifying minimum exposure requirement. This increase following resting, local heat stress which does not alter core body temperature offers mechanistic insight as elevations in blood flow and shear stress provide a non-core body temperature dependent HSP response that parallels transcription of angiogenic markers (Gao., et al.) Not all experimental work has observed changes in HSP following passive heating. Leg immersion in hot water at $45^{\circ} \mathrm{C}$ for $60 \mathrm{~min}$, eliciting an increased intramuscular temperature of $>39^{\circ} \mathrm{C}$, did not affect muscle HSPs (HSP70, HSC70, HSP60, HSP27, alphaB-crystallin) in healthy young humans [39]. It should be noted that this null-observation came $48 \mathrm{~h}$ following, rather than immediately after heating, which may provide a rationale for the response. These data share commonality with responses observed elsewhere in relation to HSP70 and HSP27 stasis $24 \mathrm{~h}$ following $\sim 80 \mathrm{~min}$ of heating at $\sim 49^{\circ} \mathrm{C}$ [37] to increase intramuscular temperature also to $>39^{\circ} \mathrm{C}$, suggesting that the dose of heat therapy may be an important driver of HSP response or that the inconsistent timing of differential tissue sampling are experimental artifacts impacting current understanding.

Examination of extracellular changes in HSP70 during acute exercise-heat stress in humans has identified that the endogenous requirement for extracellular HSP70 release (at the cessation of exercise) may be a core body temperature mean of $>38.5^{\circ} \mathrm{C}$ (peak of $39.2^{\circ} \mathrm{C}$ ) for $56 \mathrm{~min}$, alongside moderate exercising intensities [44]. Although changes may occur more rapidly (within $27 \mathrm{~min}$ ) if exercise intensities are higher). Both the change in and final core body temperature attained are relevant to extracellular HSP70 release) and indicate achieving substantial elevations in thermal parameters is important when administering exercise-heat exposures to increase thermotolerance in whole-body models.

\section{Chronic effects of body warming on heat shock proteins}

The HSP responses to exercise-heat acclimation have been reviewed previously, with an acknowledgment that the intervention is an effective means to augment cellular thermotolerance, which may subsequently protect vital organs from deleterious effects of heat stress in humans [6]. An internal temperature threshold for intracellular HSP70 induction may exist, though it is also possible that this response occurs once a certain variation of internal, whole-body temperature is reached alongside additional stressors. For example, during a 10-day heat acclimation period, demonstrated the largest changes in post-exercise intracellular HSP70 when a core body temperature $>39.0^{\circ} \mathrm{C}$ was achieved. In contrast [28] reported no change in HSP70 in response to a lower core body temperature (mean maximum of $\sim 38.5^{\circ} \mathrm{C}$ ) following 10 days of heat acclimation. HSP70 and HSP90 mRNA transcription occur at a series of core body temperature thresholds during $90 \mathrm{~min}$ of exercise-heat stress (mean $37.6-38.2^{\circ} \mathrm{C}$; peak $38.1-39.1^{\circ} \mathrm{C}$ ) [44] therefore the dose of heat stress to elicit translation may be greater than that required to elicit transcription.

In human whole-body passive heating models, such as hot water immersion of $>60$ min whereby core body temperature is increased, higher extracellular HSP70 concentration [37], and intracellular HSP70 changes, have been reported. The beneficial response once again is not unanimous, with others reporting chronic change (following 2 weeks of repeated therapy) in intracellular HSP70 after 45 - 60 minutes of passive heat therapy). In a similar manner to that described for local heating, the dose of stress may be important given the lack of change in studies using 60 minutes heating, in comparison to those who do report an acute increase in intracellular HSP70 following $120 \mathrm{~min}$ heating in $39^{\circ} \mathrm{C}$ water [42].

Intracellular HSP70 and HSP90 levels in peripheral blood mononuclear cells (PBMC) are increased after 6-10 days of long term exercise-heat acclimation [15]. The two larger HSPs appear to be related with regards to exercise-heat stress changes, as an HSP70 increase of $\sim 21 \%$ was correlated with HSP90 increases of $\sim 18 \%$ ). In vitro analysis of PBMC obtained from 10-day exerciseheat acclimated individuals exhibit greater blunting of the HSP response to heat shock of $43^{\circ} \mathrm{C}$ for $60 \mathrm{~min}$ (compared to unaccli- 
mated). This blunted pre-post response is indicative of increased thermotolerance and/or cellular protection from stress, likely due to increased basal intracellular HSP content and appears directly related to the degree of physiological heat acclimation (lower core temperature), thus the adapted individual/cell experiences lesser relative stress at the same absolute temperature [9].

The notion of individual differences in the responses to local heat therapy has been highlighted in a study observing that $24 \mathrm{~h}$ following $40 \mathrm{~min}$ of heat treatment (diathermy followed by heat packs), female subjects significantly increased HSP70 (+58\%) and phosphorylation of HSP27 (+100\%) content compared to the untreated leg). In comparison, male subjects had non-significant increases in HSP70 (+35\%) and HSP27 phosphorylation (+32\%) within skeletal muscle. These sex-specific responses are intriguing and warrant future investigation given no differences in Hsp70 mRNA have been reported during isothermic heat acclimation and were not reported in the mixed-sex cohort undertaking passive heating described above [37].

\section{Central and peripheral effects of body warming}

The administration of heat therapy and heat acclimation may provide additional benefits in the context of neurodegenerative diseases in relation to skeletal muscle function, cerebral blood flow, and metabolic health. Adverse reductions in strength and lean body mass are symptoms of neurodegenerative diseases, including Alzheimer's disease [21,22], Parkinson's disease [11] and Amyotrophic Lateral Sclerosis. While various mechanisms are at play, muscle atrophy and decreased strength likely owe to symptom-influenced reductions in physical activity, along with central and peripheral nervous system changes that limit muscle activation. Furthermore, reduced cerebral blood flow and poor metabolic health profiles may also be related to disease progression [13,30,35]. As there is a potential benefit to be gained, the below reviews the current developing understanding of the acute and chronic effects of elevated temperature on skeletal muscle function, cerebral blood flow, and markers of metabolic health.

\section{Skeletal muscle function}

Increases in skeletal muscle temperature have long been accepted to improve acute muscle force, power and contractility $[12,29]$. Contrastingly, the inverse relationship between high core body temperature and muscle torque, muscle recruitment patterns, and voluntary activation is also documented. Importantly, however, these outcomes are most often viewed with a short-term lens, with limited understanding of the effects of time or repeated heat exposures on skeletal muscle in humans [15].

Passive heating has been reported in experimental designs examining effects on exercise-induced muscle damage ecovery from muscle injury and immobilization and muscle hypertrophy in animal models. The rationale for passive heat application relates to the altered cascade of inflammation and HSP expression that interact with mitochondrial biogenesis and muscle growth increases in wet muscle mass and protein content in rat soleus muscle have been described 7 days after a $60 \mathrm{~min}$ exposure to a $41-42^{\circ} \mathrm{C}$ heat chamber. Further observed higher muscle mass/body weight ratios in rats following 30 days of acclimation to $34^{\circ} \mathrm{C}$ environmental heat compared to a $24^{\circ} \mathrm{C}$ control. Similarly, in healthy men, saw an increased cross-sectional area of fibers in the vastus lateralis (8.3\%) using a steam-generating sheet applied to the quadriceps muscle for $8 \mathrm{~h} \cdot$ day $^{-1}$ and 4 days week across a 10 -week intervention. Collectively, it might be concluded that passive heating could support cell proliferation and facilitate muscle hypertrophy). Such outcomes would be particularly beneficial to those experiencing neurodegenerative disease, particularly as passive heating appears also to attenuate human skeletal muscle atrophy [29].

Most pertinent from a translational perspective, improved strength has been demonstrated to couple the increased skeletal muscle growth after passive heating. Higher isometric knee extensor torque (5.8\%) was achieved after 10 weeks of heat stress, which the authors explained as potentially relating to the increase of myonuclear number also found a $4 \%$ increase in knee extensor strength in the non-heated leg. While the contralateral effects of unilateral resistance training are acknowledged [40], this phenomenon may imply that there are central nervous system effects of chronic passive heating. A potential role of circulating factors has also been proposed). The site(s) of possible neural adaptation explaining cross-education remain unclear. However, it is conceivable that adaptations could occur at the spinal and/or cortical level considering the noted decline in descending motor drive during acute episodes of hyperthermia. It seems that higher body temperature acutely impairs somatosensory processing, though how adaptation to passive heating might affect neural activity in healthy or diseased states in thermoneutral conditions remains to be elucidated. Regardless, adaptation to passive heat therapy is promising, particularly in a rehabilitation setting and for those with neuro- 
degenerative diseases, as acute stress may increase motor cortex excitability and augment motor skill acquisition [42].

\section{Cerebral blood flow}

Another avenue by which passive heating may have a therapeutic effect is through improved cerebral blood flow. Reduced cerebral blood flow and dysfunction in the blood-brain barrier have been identified in neurodegenerative diseases, including Alzheimer's and Parkinson's $[5,30]$. Both motor and cognitive impairments have been associated with poor perfusion in several brain regions in Parkinson's disease). Similarly, overall and regional cerebral blood flow reductions have been associated with cognitive decline in mild cognitive impairment and Alzheimer's disease. For these reasons, the vasculature supplying blood to and across the brain are also relevant targets to examine the beneficial adaptations of passive heat therapy for neurodegenerative diseases.

Several vascular adaptations attributable to heat therapy have been reported. These have included improved flow-mediated dilation [18], increased pulse wave velocity (an index of arterial stiffness) [18], reduced carotid intima thickness [18] improved capillarization and subsequently enhanced systemic blood pressure profiles $[4,18]$. Mechanistically, HSP27 has been shown to reduce intimal hyperplasia [27], with greater carotid intima thickness associated with reduced cerebral blood flow. Undesirable vascular hypertrophy can also be mitigated against via HSP70 associated inhibition of Angiotension II), with HSP90 conferring a more general adaptation aligned to the stabilization of the vascular endothelial growth factor (VEGF) upstream target hypoxia-inducible factor-1 (HIF-1 $\alpha$ )), and elevated endothelial nitric oxide production and improved the stabilization and bioavailability of endothelial nitric oxide synthase [9]. Collectively these vascular adaptations observed in passive heating interventions have the potential to maintain cerebral blood flow and blood-brain barrier function, outcomes which may have beneficial effects for cognitive function in Alzheimer's and Parkinson's diseases.

\section{Metabolic health}

Markers of metabolic health may also play a role in the pathogenesis of neurodegeneration in Alzheimer's disease. The neurodegeneration seen in Alzheimer's disease has been linked with impaired cerebral insulin signaling and glucose metabolism [13]. The loss of protein homeostasis ( $\mathrm{A} \beta$ accumulation and tau hyperphosphorylation), synaptic degeneration, and neural dysfunction have been associated with these impairments to normal metabolic processes [13]. Recent research has therefore proposed therapeutic interventions to improve insulin signaling and reduce insulin resistance. Interestingly, heat therapy has been proposed for people with type II diabetes, and early studies have highlighted a reduction in fasting plasma glucose following repeated hot water bath immersions over 3 weeks. Furthermore, chronic heat therapy interventions have improved glucose tolerance and insulin sensitivity in women with polycystic ovary syndrome who experience obesity and metabolic dysfunction [34]. Therefore, the effects of heat therapy on metabolic health are another potential avenue of therapeutic benefit for people with neurodegenerative disease.

\section{Summary}

For individuals who do not experience these important physiological stressors through habitual activity and exercise, heat therapy may provide a vehicle to achieve a range of health and physiological benefits (Figure 1). Much remains to be understood with regards to the mechanisms and stimuli required to elicit the desired increases in relevant HSPs following heat therapy, in addition to further quantifying the magnitude of importance of these responses. Additionally, the potential to improve muscular function, cerebral blood flow, and markers of metabolic health offer significant benefit for people with a neurodegenerative disease by improving their quality of life and reducing disease severity. Part of the present ambiguity results from the utilization of different methods, e.g., heating technique, heating duration and magnitude, tissue sample site and time points, across experimental studies. Despite the need for further clarity regarding the mechanistic underpinnings and best practice implementation, the opportunities to investigate a tolerable heat therapy model in a relevant target population exists and should be encouraged.

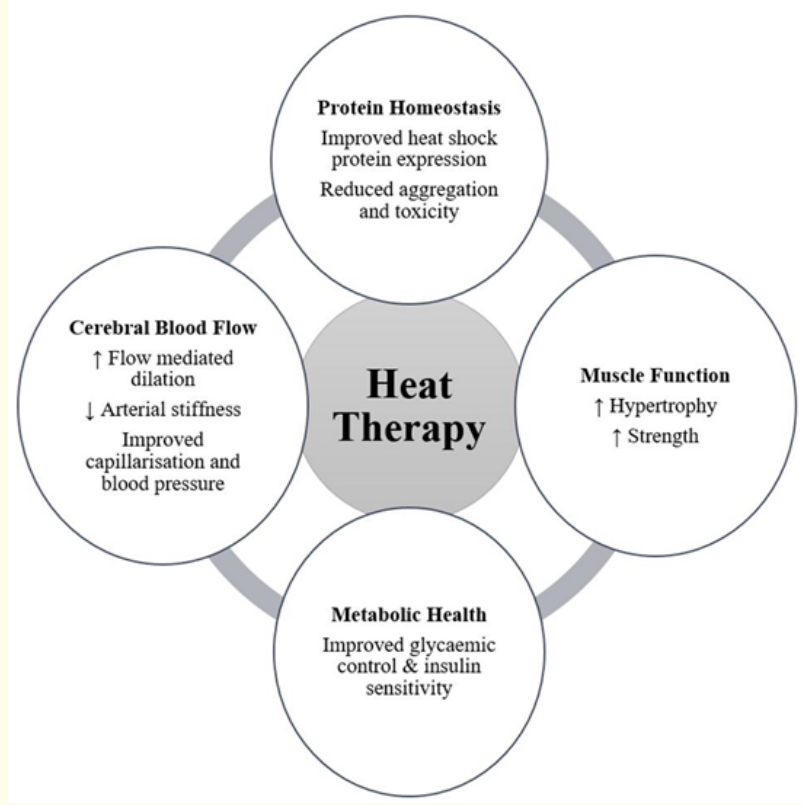

Figure 1: Potential benefits arising from heat therapy for people with neurodegenerative disease ( $\uparrow$ symbolizes an increasing effect; $\downarrow$ symbolizes a decreasing effect). 
Body warming in people with neurodegenerative diseases

Evidence for a potential benefit

At present, the authors are not aware of any studies that have directly assessed the effects of active or passive body warming on HSP release, and disease severity or progression, in people with neurodegenerative diseases. Therefore, this review will draw on findings from epidemiological studies, and indirect studies of other interventions such as exercise, that have shown benefits among people with neurodegenerative diseases.

Habitual body warming, through sauna bathing or exercise, has been shown to reduce the risk of developing neurodegenerative diseases. Regular passive heating has been associated with a reduced risk of developing neurodegenerative diseases, including Alzheimer's). Men participating in sauna bathing 2-3 or 4-7 times per week had a 0.80 and 0.35 hazard ratio for developing Alzheimer's disease compared to men who sauna once per week or less). Regular exercise also has a protective effect on the risk of developing Alzheimer's and Parkinson's diseases). Consistent and frequent participation in moderate to vigorous physical activity was found to reduce the risk of Parkinson's disease by up to 40\%). Similarly, exercising three or more times per week is associated with a lower incidence rate of dementia and Alzheimer's. While these studies do not elucidate the potential mechanisms by which a protective effect is elicited, it does lend anecdotal evidence toward the expression of HSP through regular body warming as a distinct possibility.

Further to a preventative effect, exercise has been described as having a restorative effect on the neurodegeneration observed in Alzheimer's and Parkinson's diseases). Clinical and epidemiological studies have provided evidence supporting the conclusion that exercise has therapeutic value by reducing the symptoms and slowing disease progression). Moderate to high-intensity aerobic exercises, such as treadmill walking or assisted cycling, are recommended for improved motor and cognitive function in Parkinson's disease [3,35]. High-intensity treadmill exercise of $30 \mathrm{~min}$ at a target heart rate prevented Parkinson's disease progression, compared to moderate intensity and control. Furthermore, interval exercises (alternating periods of low and high intensity assisted cycling) have shown positive improvements in functional ability in Parkinson's patients. Immersion in warm water $\left(33^{\circ} \mathrm{C}\right)$ for $50 \mathrm{~min}$ while performing dual-task exercises (combining physical movements with cognitive tasks) was found to improve functional mobility (timed up and go, and five-time sit-to-stand) among people with Parkinson's disease following 3 months of twice-weekly exposures [25].

Exercise interventions have also had significant effects on slowing the progression of Alzheimer's disease. Over 1 year, people with Alzheimer's disease who participated in twice-weekly 1-h exercise sessions showed reduced rates of decline in measures of functional independence and physical performance, compared to control participants. In a similar intervention, the ability to perform activities of daily living declined significantly slower in people with Alzheimer's disease performing twice-weekly exercise programs. A 6-month walking program has also shown people with Alzheimer's disease to be able to maintain cognitive function on the Mini Mental State Exam (MMSE), compared to significant declines in those not exercising). Overall, there is compelling evidence that exercise improves motor and cognitive function in neurodegenerative disease and it is therefore recommended by clinicians. However, exercise and body warming interventions should consider the difficulties in performing such activities for these populations (section "Considerations for Heat Therapy for People With Neurodegenerative Disease").

The studies of moderate to high-intensity exercise among neurodegenerative disease populations provide circumstantial evidence that the assumed body warming experienced may be contributing to the beneficial effects observed. While there may be many avenues by which exercise and body warming promote improved health and function, the role of body temperature elevation, thermoregulatory responses, and HSP expression have been overlooked in these experiments. Given the growing body of evidence that supports the expression of HSPs as therapeutic targets for Alzheimer's and Parkinson's diseases (section “Heat Shock Proteins As a Therapeutic Target"), there is a clear need for future investigations of passive heating to monitor thermoregulation and HSP responses in people with these neurodegenerative diseases.

Considerations for heat therapy for people with neurodegenerative disease

An important consideration in conducting heat acclimation and heat therapy for older adults and clinical populations will be how their impairments or any co-morbidities may affect their ability to perform and tolerate these interventions. Firstly, their disease severity may impair their physical ability to perform movements effectively and safely [21]. Secondly, neurodegeneration may cause 
deficits in thermoregulatory processes. In Parkinson's disease, neural degeneration in higher-order brain centers including the hypothalamus is associated with impaired sudomotor function which in turn may influence their tolerance to body warming.

Neurodegenerative diseases such as Parkinson's and Motor Neuron Disease primarily affect motor control. As the diseases progress, motor function deteriorates resulting in impaired gait and balance and an increased risk of falls. While Alzheimer's is usually associated with cognitive impairments, significant motor impairments are also associated with this disease [21]. Therefore, exercise and heat acclimation interventions should consider the level of impairment of their target population and how the risk of falls and injury may be managed. As such, in these populations where movement is limited, passive heat therapy may be an achievable alternative to exercise interventions.

The autonomic nervous system, responsible for thermoregulation, can exhibit deficits in neurodegenerative diseases, specifically in the thermoeffector responses of sweating and skin blood flow. Abnormalities in the sweating response, hyperhidrosis and/or hypohidrosis, are commonly reported in Parkinson's disease and may be more prominent with increasing age and disease severity of patients. Hyperhidrosis, an absence of the sweating or reduced sweat output, may reduce effective body cooling during exercise and body warming. However, the sweating response is highly individualized, and compromised sweating in one body region may be compensated by increased sweating in other body regions. Careful attention should, therefore, be given to the rate of body warming people with Parkinson's disease may experience, and ensuring appropriate cooling strategies are available.

Cardiovascular regulation of blood pressure is also influenced by neurodegenerative disease. The expected elevation in heart rate and blood pressure may be blunted in people with Parkinson's disease, and they may also experience post-exercise hypotension [7]. Orthostatic intolerance is also reported in 10-60\% of people with Parkinson's disease. These cardiovascular impairments may influence their ability to tolerate exercise and body warming and should be considered in determining individual suitability for heat therapy and heat acclimation. The modality of passive heating may therefore also be an important factor in determining appropriate therapeutic techniques. Infrared sauna bathing has been reported to promote lower cardiovascular strain than traditional sauna tech- niques) and may be one avenue that could be investigated for suitability in these at-risk populations.

Overall, there are three key points from the scientific literature that support a proposal for a therapeutic effect of heat therapy or heat acclimation to promote HSP expression in people with neurodegenerative disease. These include, (1) Exercise, sufficient to raise core body temperature, is currently recommended for people with neurodegenerative disease (e.g., Parkinson's and Alzheimer's diseases) as it has been shown to improve their symptoms; (2) Elevated HSP levels have been identified as a therapeutic target to reduce protein aggregation and toxicity; and (3) Exercise and body warming have been shown to elevate HSP expression in healthy adults. Furthermore, heat therapy may have additional benefits for muscle function, vasculature health and cerebral blood flow, and indicators of metabolic health, which have also been implicated in the pathophysiological presentation of neurodegenerative diseases. These findings from the current scientific literature support the proposal for further investigation into the potentially beneficial adaptations for people with neurodegenerative diseases to heat therapy and heat acclimation.

Initial research is required to establish the acute effects of heat therapy and/or heat acclimation in people with neurodegenerative diseases. As these diseases may involve impairment of autonomic pathways involved in thermoregulation, research is required to elucidate the thermoeffector responses, sweating and skin blood flow, to acute heat stress and how these may affect tolerance to body warming. Alongside these outcomes HSP expression, muscular adaptations, and vasculature function responses to body warming should be measured in neurodegenerative disease populations, to determine the presence of a similar response to body warming as seen in healthy adults, and the magnitude of the response in relation to the tolerable limits of body warming. Consequent to acute observations that tolerable heat exposures promote the desirable HSP response and vascular adaptations, further investigation should then pursue the effects of chronic or repeated heat therapy and/or heat acclimation on these responses and indicators of disease severity and progression. Finally, an understanding of the dose-response relationship between frequency and intensity of body warming and improved symptomology should be determined. In concert with these research efforts, investigations of the underlying mechanisms by which HSP expression and body warming may improve neuromuscular function are warranted [29]. 


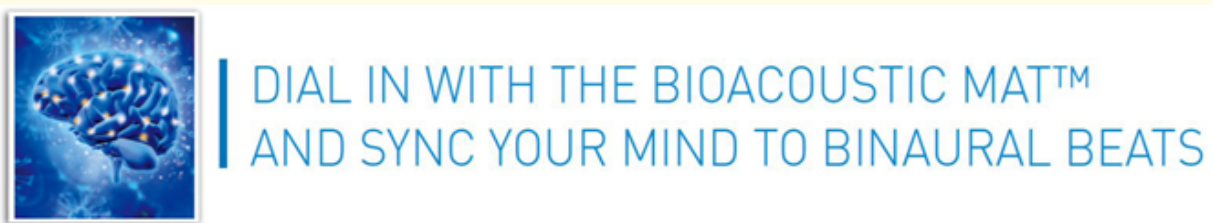

Figure $b$

If you ever found yourself tapping your feet to the beat of a song, you have the basic idea of entrainment.

It is a physics principle, where one rhythmic system falls in line with another. It is our body's way of going with the flow.

From a physics standpoint, less energy is required when one system falls in line with another. The human brain is a complex system and is made up of an immense neural network which sends and receives signals. It shapes our entire reality. The pulses are rhythmic in nature and can be temporarily subject to entrainment.

The BioAcoustic Mat's ${ }^{\mathrm{TM}}$ music is embedded with specific frequencies that coax our mind into different therapeutic states. The carefully constructed tracks stimulate healthy neural activity in the brain by controlling the number of sounds the ear hears per second and by playing a slightly different pitch in each ear. This results in our mind syncing with the rhythm of our body becoming more relaxed or energized.
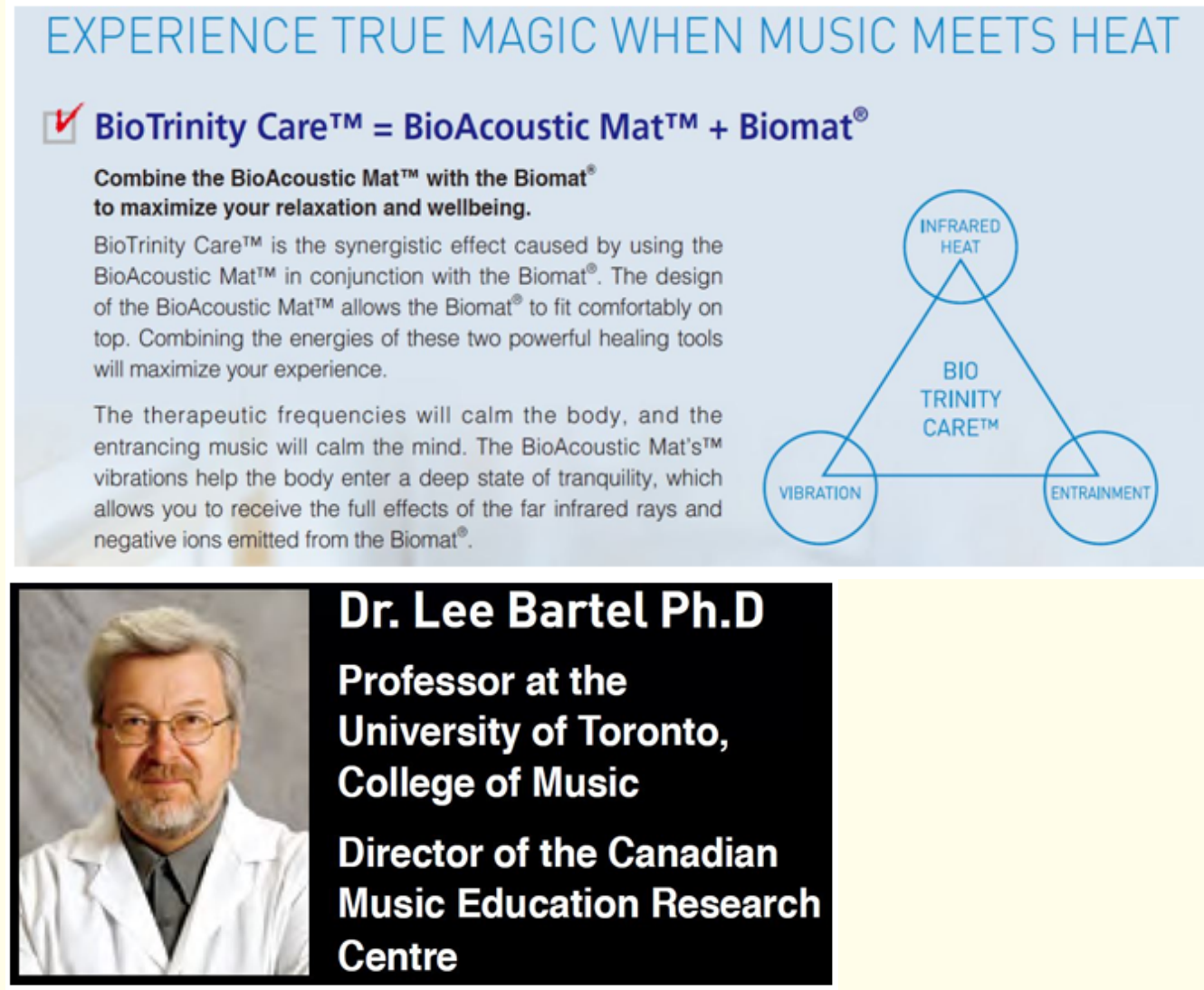

Figure c 
Dr. Bartel is a Professor Emeritus of Music and former Associate Dean for research at the University of Toronto's Faculty of Music, as well as Director of Music and Health Research Collaboratory.

Dr. Bartel has special interest in applications of music in health conditions of aging and rehabilitation. He is well known for his research and design of music for health. His primary research interest is currently in Rhythmic Sensory Stimulation (RSS) with applications for several types of pain.

Within the Canadian Music Education Research Centre, Dr. Bartel is the Coordinator of the Music Therapy and Health Special Research Interest Group.

He has also served on the Accreditation Review Board for the Canadian Association of Music Therapy, has been involved in noteworthy studies with the Centre for Health Promotion, University of Toronto, and has supervised doctoral and post-doctoral research at Bloorview Kid's Rehab, Lyndhurst Hospital, and Baycrest Hospital.

Drawing upon his many years of experience, Dr. Bartel designed the internationally best-selling Music for Your Health series from the Solitude's label, as well as Somerset Entertainment's SonicAid series. His Gold and Platinum album work has been nominated for several Juno Awards.

Today, Dr. Bartel's contributions include 16 books, multiple academic papers and publications.

The BioAcoustic Mat ${ }^{\mathrm{TM}}$ is useful for...

- $\quad$ Stress Reduction

- Entraining

- Physical and auditory stimulation

- Music Therapy

Use the BioAcoustic Mat ${ }^{\mathrm{TM}}$ in conjunction with the Biomat ${ }^{\circledR}$ for optimal performance.

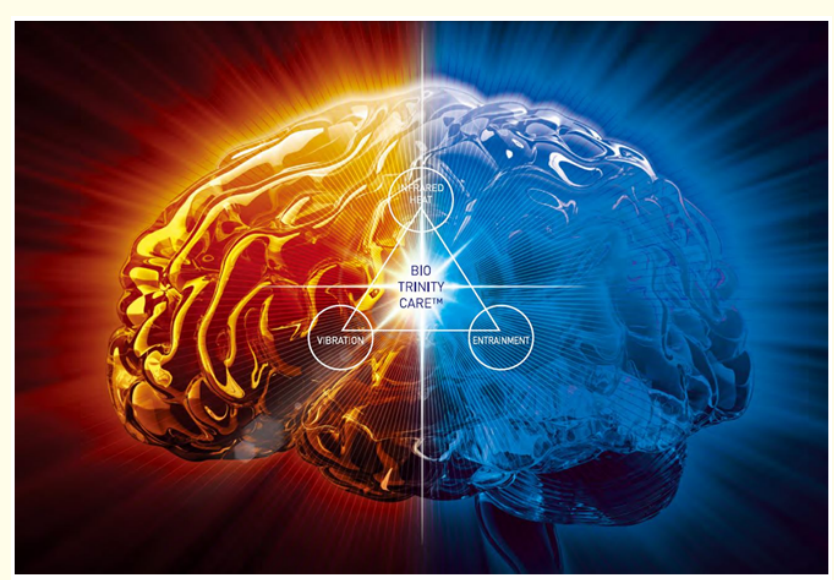

(C) RC-REM-RnL-BTC700mx FG FGC

Figure d
Rhythmic oscillatory coherence and connectivity: The origin and function of neural rhythmic oscillatory activity in the brain remains a central research question in neuroscience. Although several explanations have been proposed [1], recent work has raised important issues related to clinical application of oscillatory brain activity in neurodegenerative diseases and neurorehabilitation [2] Can oscillatory brain activity be used as diagnostic biomarker for conditions like Parkinson's disease (PD), Alzheimer's disease (AD), and depression? And can oscillatory brain activity be modulated in support of therapeutic interventions? The healthy human brain has intrinsic and constant rhythmic oscillation. Popular psychology explained the oscillatory rhythms of "brain waves" as neural indices of specific mental states, e.g. delta with sleep $(0.1-4 \mathrm{~Hz}$ ) theta with deep relaxation and creative insight $(4-8 \mathrm{~Hz})$, alpha with relaxation $(8-12 \mathrm{~Hz})$, and beta with problem solving $(12-20 \mathrm{~Hz})$. Although generally true, scientific interest is moving toward understanding the role that oscillatory rhythms may play in coordinating neural activity supporting perceptual, cognitive, and motor functions [3]. Oscillatory activity may index local neural networks from modality-specific brain areas as well as long-range neural systems that engage sensory and supra-modal brain regions (e.g., prefrontal and parietal cortices) [4]. Ward [5] proposed that consciousness is related to synchronous neural rhythms in general, but that memory processes are related to gamma $(30-50 \mathrm{~Hz})$ and theta oscillatory rhythms, whereas attention is dependent on alpha and gamma activity. His review of the literature points toward an increased connectivity between frontal and parietal cortex during memory recall from larger spectral power in gamma and theta frequency bands, with the magnitude of gamma activity modulated by the theta rhythm. This intra-brain communication through neuronal oscillatory coherence is thought to index healthy functioning of specific circuits - like memory, or movement. Although it is well accepted that brain activity related to perceptual, cognitive, and motor functions depends on widely distributed neural networks, the functional connectivity between the nodes of the networks is less well understood. Fries [6] proposed that the mechanism of neuronal communication depends on similar neuronal oscillatory activity and that communication within a local network (e.g., sensory cortex) happens mainly with coherent oscillation in the gamma frequency range (30-100 Hz). For communication between distant brain areas, the amount of local gamma oscillations is controlled by slower rhythm in the theta frequency range $(4-8 \mathrm{~Hz})$ [7].

\section{Bibliography}

1. Access Economics (Firm), and Parkinson's Australia. Living with Parkinson's Disease: Challenges and Positive Steps for the Future. Scullin: Access Economics (2007).

2. H Katsuno., et al. "Heat shock proteins in neurodegenerative diseases: pathogenic roles and therapeutic implications". International Journal of Hyperthermia 25 (2009): 647-654.

3. Ahlskog JE. "Does vigorous exercise have a neuroprotective effect in Parkinson disease?" Neurology 77 (2011): 288-294. 
4. Akerman AP., et al. "Heat therapy vs. supervised exercise therapy for peripheral arterial disease: a 12-week randomized, controlled trial". American Journal of Physiology-Heart and Circulatory Physiology 316 (2019): H1495-H1506.

5. Akinyemi RO., et al. "Vascular risk factors and neurodegeneration in ageing related dementias: Alzheimer's disease and vascular dementia". Current Alzheimer Research 10 (2013): 642-653.

6. Amorim FT., et al. "Insights into the role of heat shock proteins 72 to whole-body heat acclimation in humans". Temperature 2 (2015): 499-505.

7. Asahina M., et al. "Autonomic dysfunction in parkinsonian disorders: assessment and pathophysiology". Journal of Neurology, Neurosurgery, and Psychiatry 84 (2013): 674.

8. Auluck PK., et al. "Chaperone suppression of alpha-synuclein toxicity in a Drosophila model for Parkinson's disease". Science 295 (2002): 865-868.

9. Averna M., et al. "Functional role of HSP90 complexes with endothelial nitric-oxide synthase (eNOS) and calpain on nitric oxide generation in endothelial cells". Journal of Biological Chemistry 283 (2008): 29069-29076.

10. Bennett AF. "Thermal dependence of muscle function". American Journal of Physiology-Regulatory, Integrative and Comparative Physiology 247 (1984): R217-R229.

11. Berardelli, A., et al. "Pathophysiology of bradykinesia in Parkinson's disease”. Brain 124 (2001): 2131-2146.

12. Bergh $U$ and Ekblom B. "Influence of muscle temperature on maximal muscle strength and power output in human skeletal muscles". Acta Physiologica Scandinavica 107 (1979): 33-37.

13. Bharadwaj, P., et al. "The link between type 2 diabetes and neurodegeneration: roles for Amyloid-beta, Amylin, and Tau proteins". Journal of Alzheimer's Disease 59 (2017): 421-432.

14. Braak, H., et al. "Staging of brain pathology related to sporadic Parkinson's disease". Neurobiology Aging 24 (2003): 197-211.

15. Brazaitis M., et al. "Heat acclimation does not reduce the impact of hyperthermia on central fatigue". European Journal of Applied Physiology 109 (2010): 771-778.

16. Breen D P., et al. "Gut-brain axis and the spread of alpha-synuclein pathology: vagal highway or dead end?" Movement Disorder 34 (2019): 307-316.
17. Brunt VE., et al. "Passive heat therapy improves cutaneous microvascular function in sedentary humans via improved nitric oxide-dependent dilation". Journal of Applied Physiology 121 (2016a): 716-723.

18. Brunt VE., et al. "Passive heat therapy improves endothelial function, arterial stiffness and blood pressure in sedentary humans". Journal of Physiology 594 (2016b): 5329-5342.

19. Brunt VE., et al. "Serum from young, sedentary adults who underwent passive heat therapy improves endothelial cell angiogenesis via improved nitric oxide bioavailability". Temperature 6 (2019): 169-178.

20. Brunt VE., et al. "Passive heat therapy protects against endothelial cell hypoxia-reoxygenation via effects of elevations in temperature and circulating factors". Journal of Physiology 596 (2018): 4831-4845.

21. Buchman A and Bennett DA. "Loss of motor function in preclinical Alzheimer's disease". Expert Review on Neurotherapy 11 (2011): 665-676.

22. Burns J M., et al. "Reduced lean mass in early Alzheimer disease and its association with brain atrophy". Archives of Neurology 67 (2010): 428-433.

23. Cano-de-la-Cuerda R., et al. "Is there muscular weakness in Parkinson's disease?" American Journal of Physical Medicine and Rehabilitation 89 (2010): 70-76.

24. Carman A., et al. "Chaperone-dependent Neurodegeneration: a molecular perspective on therapeutic intervention". Journal of Alzheimers Disease and Parkinsonism (2013): 007.

25. Castro D M., et al. "The economic cost of Alzheimer's disease: family or public health burden?" Dementia and Neuropsychologia 4 (2010): 262-267.

26. Ciechanover, A., et al. "Protein quality control by molecular chaperones in Neurodegeneration". Frontiers in Neuroscience 11 (2017): 185.

27. Connolly EM., et al. "Pharmacological induction of HSP27 attenuates intimal hyperplasia in vivo". European Journal of Vascular and Endovascular Surgery 25 (2003): 40-47.

28. Danzer KM., et al. "Heat-shock protein 70 modulates toxic extracellular alpha-synuclein oligomers and rescues trans-synaptic toxicity". FASEB Journal 25 (2011): 326-336. 
29. Davies CT and Young K. "Effect of temperature on the contractile properties and muscle power of triceps surae in humans". Journal of Applied Physiology 55 (1983): 191-195.

30. de la Torre JC. "Is Alzheimer's disease a neurodegenerative or a vascular disorder? Data, dogma, and dialectics". Lancet Neurology 3 (2004): 184-190.

31. De Marinis M., et al. "Alterations of thermoregulation in Parkinson's disease”. Functional Neurology 6 (1991): 279-283.

32. Dodd SL., et al. "Hsp27 inhibits IKKbeta-induced NF-kappaB activity and skeletal muscle atrophy". FASEB Journal 23 (2009): 3415-3423.

33. Dorsey ER., et al. "Global, regional, and national burden of Parkinson's disease, 1990-2016: a systematic analysis for the global burden of disease study 2016". Lancet Neurology 17 (2018): 939-953.

34. Ely BR., et al. "Heat therapy improves glucose tolerance and adipose tissue insulin signaling in polycystic ovary syndrome". American Journal of Physiology-Endocrinology and Metabolism 317 (2019): E172-E182.

35. Evans CG., et al. "Heat shock proteins 70 and 90 inhibit early stages of amyloid beta- (1-42) aggregation in vitro". Journal of Biological Chemistry 281 (2006): 33182-33191.

36. Evens A and Clark C. "Does assisted cycling improve function in those with Parkinson's disease?" Physical Therapy Reviews 22 (2017): 243-253.

37. Faulkner SH., et al. "The effect of passive heating on heat shock protein 70 and interleukin-6: a possible treatment tool for metabolic diseases?" Temperature 4 (2017): 292-304.

38. Findley LJ. "The economic impact of Parkinson's disease”. Parkinsonism and Related Disorders 13 (2007): S8-S12.

39. Fontaine SN., et al. "Neurodegeneration and the heat shock protein 70 machinery: implications for therapeutic development". Current Topics in Medicinal Chemistry 16 (2016): 27412752.

40. Frazer AK., et al. "Determining the potential sites of neural adaptation to cross-education: implications for the cross-education of muscle strength". European Journal of Applied Physiology 118 (2018): 1751-1772.

41. Galton CJ., et al. "Atypical and typical presentations of Alzheimer's disease: a clinical, neuropsychological, neuroimaging and pathological study of 13 cases". Brain 123 (2000): 484-498.
42. Gao X., et al. "Human Hsp70 disaggregase reverses parkinson's-linked alpha-synuclein Amyloid fibrils". Molecular Cell 59 (2015): 781-793.

43. Gibson OR., et al. "Extracellular Hsp72 concentration relates to a minimum endogenous criteria during acute exercise-heat exposure". Cell Stress Chaperones 19 (2014): 389-400.

44. Gibson OR., et al. "Isothermic and fixed-intensity heat acclimation methods elicit equal increases in Hsp72 mRNA". Scandinavian Journal of Medicine Science 25 (2015a): 259-268.

\section{Assets from publication with us}

- Prompt Acknowledgement after receiving the article

- Thorough Double blinded peer review

- Rapid Publication

- Issue of Publication Certificate

- High visibility of your Published work

Website: www.actascientific.com/

Submit Article: www.actascientific.com/submission.php

Email us: editor@actascientific.com

Contact us: +919182824667 